\title{
Improving Vitamin D testing and supplementation in children with newly-diagnosed cancer: A Quality Improvement Initiative at Rady Children's Hospital San Diego
}

Ksenya Shliakhtsitsava ${ }^{1}$, Erin Stucky Fisher ${ }^{2}$, Erin Trovillion ${ }^{3}$, Kelly Bush ${ }^{2}$, Dennis Kuo ${ }^{4}$, Ron Newfield ${ }^{2}$, Courtney Thornburg ${ }^{5}$, William Roberts ${ }^{2}$, and Paula Aristizabal ${ }^{6}$

${ }^{1}$ UT Southwestern Medical

${ }^{2}$ University of California San Diego

${ }^{3}$ Atrium Health

${ }^{4}$ University of California San Diego School of Medicine

${ }^{5}$ Rady Children's Hospital-San Diego

${ }^{6}$ Univeristy of California San Diego, Rady Children's Hospital San Diego

January 12, 2021

\begin{abstract}
Background: Vitamin D deficiency and insufficiency have been associated with poorer health outcomes. Children with cancer are at high risk for Vitamin D deficiency and insufficiency. At our institution, we identified high variability in Vitamin D testing and supplementation in this population. Of those tested, $65 \%$ were Vitamin D deficient/insufficient. We conducted a quality improvement (QI) initiative with aim to improve Vitamin D testing and supplementation among children aged 2-18 years old with newly-diagnosed cancer to [?] $80 \%$ over 6 months. Methods: An inter-professional team reviewed baseline data, then developed and implemented interventions using Plan-Do-Study-Act (PDSA) cycles. Barriers were identified using QI tools, including lack of automated triggers for testing and inconsistent supplementation criteria and follow-up testing post-supplementation. Interventions included an institutional Vitamin D guideline, clinical decision-making tree for Vitamin D deficiency, insufficiency and sufficiency, electronic medical record triggers, and automated testing options. Results: Pre-intervention: $\mathrm{N}=26$ patients, four (15\%) had baseline Vitamin D testing; two (8\%) received appropriate supplementation. Post-intervention: $\mathrm{N}=33$ patients; 32 (97\%) had baseline Vitamin D testing; 33 (100\%) received appropriate supplementation and completed follow-up testing timely (6-8 weeks post-supplementation). Change was sustained over 24 months. Conclusions: We achieved and sustained our aim for Vitamin D testing and supplementation in children with newly-diagnosed cancer through inter-professional collaboration of hematology/oncology, endocrinology, hospital medicine, pharmacy, nursing, and information technology. Future PDSA cycles will address patient compliance with Vitamin D supplementation and impact on patients' Vitamin D levels.
\end{abstract}

Improving Vitamin D testing and supplementation in children with newly-diagnosed cancer: A Quality Improvement Initiative at Rady Children's Hospital San Diego

Ksenya Shliakhtsitsava MD MAS ${ }^{1-2}$, Erin Stucky Fisher MD ${ }^{3}$, Erin M. Trovillion MD ${ }^{1,4}$, Kelly Bush MD ${ }^{1,5}$, Dennis John Kuo MD MS${ }^{1}$, Ron S. Newfield $\mathrm{MD}^{6}$, Courtney D. Thornburg MD MS ${ }^{1,3}$, William Roberts $\mathrm{MD}^{1,5}$, Paula Aristizabal MD MAS ${ }^{1,5,7}$

1 Department of Pediatrics, Division of Pediatric Hematology/Oncology, University of California, San Diego/Rady Children's Hospital San Diego, San Diego, CA

${ }^{2}$ Now with Department of Pediatrics, Division of Pediatric Hematology/Oncology, University of Texas Southwestern, Dallas, TX 
3 Department of Pediatrics, Division of Pediatric Hospital Medicine, University of California, San Diego/Rady Children's Hospital San Diego, San Diego, CA

${ }^{4}$ Now with Department of Pediatrics, Division of Pediatric Hematology/Oncology, Atrium Health, Levine Children's Cancer and Blood Disorders, Charlotte, NC

${ }^{5}$ Quality Improvement Committee, Division of Pediatric Hematology/Oncology, Rady Children's Hospital San Diego, San Diego, CA

6 Department of Pediatrics, Division of Pediatric Endocrinology, University of California San Diego/Rady Children's Hospital San Diego, San Diego, CA

7 Population Sciences, Disparities and Community Engagement, University of California San Diego Moores Cancer Center, La Jolla, CA

\section{Corresponding author:}

Ksenya Shliakhtsitsava, MD, MAS

5323 Harry Hines Blvd.

Dallas, TX 75390

Phone: 214-456-5815

Fax: 214-648-3122

Email: Ksenya.Shliakhtsitsava@UTSouthwestern.edu

Abstract word count: 237

Main Text word count: 3,127

Number of Tables: 0

Number of Figures: 5

Supporting Information files: 0

Running title: Vitamin D testing and supplementation

Keywords: pediatric cancer, Vitamin D deficiency, Vitamin D supplementation, quality improvement, clinical guidelines

Abbreviations key:

\begin{tabular}{ll}
\hline Abbreviation & Word \\
\hline BPA & Best Practice Advisory \\
CAR & Chimeric antigen receptor \\
ng/ml & Nanogram/milliliter \\
PDSA & Plan-Do-Study-Act \\
QI & Quality Improvement \\
UVB & Type B ultraviolet \\
\hline
\end{tabular}

\section{Previously presented at (poster presentations):}

Improving the approach for Vitamin D diagnostic testing and supplementation in newly diagnosed pediatric cancer patients at Rady Children's Hospital San Diego. Pediatrics Research Symposium, San Diego, January 2017.

You Can't Find What You Don't Seek: Vitamin D Testing and Treatment in Pediatric Cancer Patients. 
PAS, San Francisco, May 2017.

\begin{abstract}
Background : Vitamin D deficiency and insufficiency have been associated with poorer health outcomes. Children with cancer are at high risk for Vitamin D deficiency and insufficiency. At our institution, we identified high variability in Vitamin D testing and supplementation in this population. Of those tested, $65 \%$ were Vitamin D deficient/insufficient. We conducted a quality improvement (QI) initiative with aim to improve Vitamin D testing and supplementation among children aged 2-18 years old with newly-diagnosed cancer to [?] $80 \%$ over 6 months.

Methods: An inter-professional team reviewed baseline data, then developed and implemented interventions using Plan-Do-Study-Act (PDSA) cycles. Barriers were identified using QI tools, including lack of automated triggers for testing and inconsistent supplementation criteria and follow-up testing post-supplementation. Interventions included an institutional Vitamin D guideline, clinical decision-making tree for Vitamin D deficiency, insufficiency and sufficiency, electronic medical record triggers, and automated testing options.
\end{abstract}

Results: Pre-intervention: N=26 patients, four (15\%) had baseline Vitamin D testing; two (8\%) received appropriate supplementation. Post-intervention: N=33 patients; 32 (97\%) had baseline Vitamin D testing; $33(100 \%)$ received appropriate supplementation and completed follow-up testing timely (6-8 weeks postsupplementation). Change was sustained over 24 months.

Conclusions: We achieved and sustained our aim for Vitamin D testing and supplementation in children with newly-diagnosed cancer through inter-professional collaboration of hematology/oncology, endocrinology, hospital medicine, pharmacy, nursing, and information technology. Future PDSA cycles will address patient compliance with Vitamin D supplementation and impact on patients' Vitamin D levels.

\title{
Manuscript word count 3,124
}

\section{Introduction}

Vitamin D plays an important role in calcium homeostasis and bone health. ${ }^{1,2}$ Large ecological studies have suggested a link between Vitamin D deficiency and low UVB irradiation with increased cancer incidence and mortality. ${ }^{3,4}$ These studies highlight a broader role for Vitamin D in the human body than previously believed, with effects on immune function, metabolism ${ }^{5,6}$ and cancer pathophysiology. ${ }^{7,8}$

Adequate calcium and Vitamin D levels are important for growing children and essential for adult bone health. Adequate calcium and Vitamin D intake, in conjunction with adequate physical activity, are recommended for cancer survivors, as well as for the general population. Children diagnosed with acute lymphoblastic leukemia are treated with systemic corticosteroids and have increased rates of skeletal complications such as osteoporosis and vertebral fractures during and after their treatment. ${ }^{9}{ }^{10}$ Additionally, suboptimal Vitamin D levels have been associated with lower survival rates after stem-cell transplantation ${ }^{11}$ and in patients with Hodgkin Lymphoma. ${ }^{8}$

Data on Vitamin D deficiency and insufficiency among children with newly-diagnosed cancer are very limited. Clinical research and Quality Improvement (QI) initiatives aimed at improving Vitamin D status in this population are lacking. We have previously reported that Vitamin D deficiency and insufficiency are common in children with newly-diagnosed cancer. Hispanic patients, females and older children were at higher risk for Vitamin D deficiency and insufficiency at our institution. ${ }^{12}$

At the University of California San Diego and Rady Children's Hospital San Diego, we noted high variability in Vitamin D testing, supplementation and follow-up testing post-supplementation for children with newlydiagnosed cancer. We conducted a QI initiative from November 1, 2015 to June 30, 2016 with the global aim to improve overall bone health among children with newly-diagnosed cancer by standardizing Vitamin D testing and supplementation.

\section{Methods}




\section{Clinical Setting and Electronic Medical Record}

This study was conducted at the University of California San Diego and Rady Children's Hospital San Diego. Rady Children's Hospital is a large tertiary academic institution. Pediatric cancer care is provided at the Peckham Center for Cancer and Blood Disorders, the largest pediatric cancer care facility in San Diego, Riverside and Imperial counties, providing care for children and adolescents with blood disorders and cancer diagnoses in a region that includes around one million children. Approximately 260 new cases of pediatric cancer are diagnosed annually at our institution. Most patients require treatment with chemotherapy, radiation, immunotherapy, stem-cell transplant, or a combination of these modalities.

During clinic visits and hospital admissions, patients with cancer are seen either by advance practice providers, resident and fellow physicians with a supervising pediatric hematology/oncology attending physician acting as the preceptor, or solely by an attending physician. All clinical documentation is completed electronically in a commercial electronic medical record (EMR) [Epic ${ }^{\circledR}$ Systems Corporation, Verona, Wisconsin], which was implemented six years prior to the beginning of our QI initiative.

\section{Target oncology population}

We previously conducted a retrospective research study to assess Vitamin D status and its socio-demographic and clinical correlates in 163 children with cancer, using 25-hydroxy vitamin D (25(OH)D) concentrations. Based on the Endocrine Society guidelines, ${ }^{13}$ we found that $65 \%$ of the patients with newly-diagnosed cancer had low Vitamin D levels. Fifty-two patients (32\%) were Vitamin D deficient, and $53(33 \%)$ were insufficient. Age over 10 years, Hispanic ethnicity, and female sex were significantly associated with lower $25(\mathrm{OH}) \mathrm{D}$ concentration at diagnosis. ${ }^{12}$ Based on these research findings, we initiated our QI initiative in November 2015. Prior to the start of the project, our institution did not have a written guideline for Vitamin D testing, supplementation, and follow-up testing post-supplementation in children with newly-diagnosed cancer. Testing for Vitamin D and supplementation was done at the discretion of the treating oncology provider. Our QI initiative included children with newly-diagnosed cancer 2-18 years of age who needed treatment with chemotherapy and/or radiation.

\section{QI Team and Specific Measurable Achievable Relevant and Timely (SMART) Aim}

After reviewing the data from our retrospective study revealing high prevalence of Vitamin D deficiency and insufficiency, we assembled an inter-professional QI team.

Our QI team followed the Institute for Healthcare Improvement's Model for Improvement ${ }^{14}$ and the QI leader completed formal QI training. The QI leader was a pediatric Hematology/Oncology physician who worked closely with the team that included physician champions, nursing staff, QI advisors, and content experts from pediatric hematology/oncology, hospital medicine, pediatric endocrinology and information technology (IT).

In person QI team meetings were held biweekly beginning with the planning phase and throughout the project to address interventions, discuss unexpected observations and problems encountered, review study data and decide on interventions to be implemented.

The entire team participated in development of the Key Driver and Ishikawa diagrams (Figures $1 \& 2$ ), the SMART Aim, the decision-making tree (Figure 3) and reviewed the Plan-Do-Study-Act (PDSA) cycles. Input from other key stakeholders (physicians, advance practice providers, oncology case managers, pharmacists, inpatient and outpatient nurses) was obtained about potential barriers and facilitators to Vitamin D testing at the time of initial cancer diagnosis and proposed supplementation strategies. The QI team leader contributed approximately six hours of time each week during the first four months of the project and then approximately four hours per week during subsequent months.

Our SMART Aim was: From February 1 to June 30, 2016, compliance with institutional guidelines for Vitamin D testing and supplementation in children with newly-diagnosed cancer (aged 2-18 years old) will be [?] $80 \%$ from a baseline of $0 \%$ and sustained over subsequent months. 


\section{Interventions}

Our institution has successfully used and sustained evidence-based order sets that include clinical decisionmaking elements for other conditions. ${ }^{15}$ The team used this existing framework, QI tools, and the PDSA methodology ${ }^{14}$ to understand barriers to improvement and implement interventions. To enable rational design of interventions, the QI team identified factors that drive the Vitamin D testing, supplementation and follow-up testing post-supplementation. Potential barriers and facilitators and primary drivers are described in Ishikawa (Figure 1) and Key Driver (Figure 2) diagrams.

After considering the feasibility, evidence, and local culture, the team decided on the following interventions: 1) development of an institutional guideline for Vitamin D testing, supplementation and follow-up testing post-supplementation; 2) education on Vitamin D deficiency and insufficiency testing and supplementation for oncology providers; 3) creation and distribution of a clinical decision-making tree for Vitamin D deficiency, insufficiency, and sufficiency [Figure 3]; 4) incorporation of EMR triggers and Vitamin D automated testing options; 5) inclusion of the Vitamin D laboratory order into the pre-existing oncology order sets; and 6) creation of a Best Practice Advisory (BPA) alert to be triggered if the patient did not have recent Vitamin D testing, or if testing was done but the patient was not taking Vitamin D.

PDSA \#1: Pre-intervention assessment, Ishikawa and Key Driver diagrams, process map, and development of institutional guideline (November 1, 2015 to January 31, 2016).

Ishikawa results were compiled and analyzed, and a process map was completed. Barriers such as lack of automated triggers/reminders for testing, inconsistent criteria used for testing and supplementation, and inability to obtain follow-up testing post-supplementation were identified. Key drivers included: Physicians' knowledge about when to test and how to supplement Vitamin D; guideline and decision-making tree widely available; integration into clinical workflows and facilitated by automated triggers in EMR; and leadership awareness and support (Figure 2). In collaboration with a pediatric endocrinologist we reviewed national best practices and created an institutional guideline and clinical decision-making tree for Vitamin D testing, supplementation and follow-up testing post-supplementation (Figure 3). We utilized 25(OH)D as the most accurate way to measure Vitamin D status in our patients. The pediatric endocrinologist provided input regarding supplementation and follow-up testing post-supplementation based on the Endocrine Society Guidelines. ${ }^{13}$ We defined Vitamin D deficiency as $25(\mathrm{OH}) \mathrm{D}$ concentration of $<20 \mathrm{ng} / \mathrm{ml}$, insufficiency [?]20 and $<30 \mathrm{ng} / \mathrm{ml}$, and sufficiency [?]30 ng/ml. Based on the patient's 25(OH)D concentration, supplementation was prescribed and follow-up testing post-supplementation was completed per the clinical decision-making tree (Figure 3).

PDSA \#2: Implementation phase (February 1, 2016 to June 30, 2016).

We conducted an educational intervention focused on Vitamin D deficiency and insufficiency testing and supplementation for oncology providers (physicians and advance practice providers), nurses, case managers, and pharmacists via direct one-on-one in-person communication, structured in-person lectures and secure e-mails. The decision-making tree was distributed as hardcopy and was made widely available and posted in the inpatient unit, outpatient clinic working space, and the secure shared oncology folder online. EMR triggers and Vitamin D automated testing options were incorporated. A Vitamin D laboratory order was added into the pre-existing oncology admission order set as well as in Beacon chemotherapy treatment plans, an Epic ${ }^{(\mathrm{r})}$ oncology module for physicians and pharmacists to create treatment and supportive care plans based on standardized protocols that also allows for easy modification. A BPA alert was created that triggered if the patient had a chemotherapy treatment plan in place but did not have recent Vitamin $\mathrm{D}$ testing, or if Vitamin D testing was done but the patient was not taking Vitamin D.

In order to assess end-user value, we created a 6-question secure online de-identified survey utilizing the SurveyMonkey ${ }^{(\mathrm{r})}$ platform. The survey was administered to oncology providers after interventions were executed. The survey included the following questions: 1) What are the barriers preventing Vitamin D initial testing at the time of cancer diagnosis? (Free text response); 2) Do you use the Vitamin D supplementation decision-making tree? (Likert scale); 3) Do you find the Vitamin D supplementation decision-making tree 
helpful? (Likert scale); 4) What are the barriers preventing repeat Vitamin D testing at 6-8 weeks after Vitamin D supplementation initiation? (Free text response); 5) Do you use the Vitamin D BPA? (Likert scale); 6) Do you find Vitamin D BPA reminders helpful? (Likert scale). For questions 2, 3, 5 and 6, a traditional Likert scale with 1-5 ratings was used to assess value/usefulness, with $1=$ none, $2=$ little, $3=$ some, $4=$ moderately high, and $5=$ high. The percentage of results for "moderately high" or "high" for each question were reported.

\section{Data collection and analysis}

The QI leader identified newly-diagnosed patients through an automated new diagnosis banner in the EMR. We performed frequent monitoring of newly-diagnosed patients to ensure that all cases were captured.

Standardized definitions for Vitamin D deficiency, insufficiency and sufficiency were used. ${ }^{13}$ EMRs of patients aged 2-18 years old with newly-diagnosed cancer were reviewed, including provider notes, lab testing orders and results, and prescriptions. Rates of Vitamin D testing, supplementation and follow-up testing postsupplementation were obtained at different time points, from November 1, 2015 to January 31, 2016 (preintervention) and from February 1, 2016 to June 30, 2016 (post-intervention), and averaged over seven-day periods. To assess sustainability, we obtained data every two months until June 2018.

Process measures of testing and supplementation were chosen to assess the system improvement; supplementation was also identified as a feasible proxy for clinical outcome improvement by the team. An additional process measure of perceived utility of the decision-tree and automated triggers was chosen to assess end-user buy-in.

Weekly documentation rates were plotted on a run chart during the study phase of each PDSA cycle to identify non-random signals of change in Vitamin D testing (Figure 4) and supplementation and follow-up testing post-supplementation (Figure 5). For statistical process control, a p-control chart was used to detect special cause variation. Both the run chart and p-control chart were generated with Microsoft Excel QI Macros. To assess process measures including use and perceived utility of the decision-making tree and automated triggers, we conducted the survey described above.

\section{Ethics and Project Communication}

This QI study was reviewed and deemed to not be human subjects research and

a QI exemption waiver was granted by the University of California San Diego/Rady Children's Hospital San Diego Institutional Review Board.

The results, successes and barriers we encountered during the project were shared with the oncology providers on a regular basis. Communications included weekly updates on the progress of the initiative, monthly updates to division leadership, and annual reports to hospital leadership.

\section{Results}

Twenty-six patients were evaluated during the initial three months (PDSA \#1): 11 patients (42\%) with hematological malignancies, 15 patients (58\%) with malignant solid tumors, mean age at the time of diagnosis was 8.9 years +-5.4, 10 patients $(38 \%)$ were males and $16(62 \%)$ females. Only four (15\%) patients had baseline Vitamin D testing. Three patients had concentrations that should have required supplementation, but only two (66\%) were prescribed the appropriate supplement.

Thirty-three patients were included in the post-intervention group (PDSA \#2): 27 patients (88\%) with hematological malignancies and six patients $(12 \%)$ with malignant solid tumors, mean age at the time of diagnosis was 8.6 years +-5.2 , 19 patients $(57 \%)$ were males and $14(43 \%)$ females. Thirty-two (97\%) had baseline Vitamin D testing performed. Thirty-three (100\%) received appropriate supplementation and completed follow-up testing at the appropriate time (6-8 weeks post-supplementation). Compared to preintervention, we tested 27 more patients and identified 10 more patients. From July 2016 to June 2018, over $80 \%$ of patients had both baseline Vitamin D testing performed, received appropriate supplementation and 
completed follow-up testing post-supplementation, demonstrating sustainability over time (Figures 4 and 5). The perceived utility and use survey was completed by 16 out of 24 clinicians and perceived utility of the decision-making tree was $67 \%$, use of decision-making tree reached $83 \%$, and perceived utility of automated triggers was $86 \%$.

\section{Discussion}

Through inter-professional collaboration with hematology/oncology, endocrinology, hospital medicine, pharmacy, and IT, we successfully improved the testing, diagnosis, supplementation and monitoring of Vitamin D deficiency and insufficiency for children with newly-diagnosed cancer and demonstrated sustainability over 24 months. We achieved and sustained our aim to improve Vitamin D testing, adequate Vitamin D supplementation and accurate and timely follow-up testing post-supplementation. We exceeded our SMART Aim of [?] $80 \%$ compliance with the newly developed institutional guideline and clinical decision-tree after implementation and sustained our aim of [?] $80 \%$ over time.

Children with cancer are at high risk for Vitamin D deficiency and insufficiency due to inadequate sun exposure and treatment-related complications, such as poor diet, hepatotoxicity and/or nephrotoxicity of chemotherapeutic agents, impaired absorption due to mucositis and/or colitis, and interference of Vitamin D metabolism by glucocorticoids. Current Endocrine Society Clinical Practice Guidelines on the evaluation, treatment, and prevention of Vitamin D deficiency recommend testing patients at risk for deficiency with the measurement of serum $25(\mathrm{OH}) \mathrm{D}$ concentrations, followed by treatment with either oral Vitamin D2 or Vitamin D3 supplementation in patients found to be deficient or insufficient. ${ }^{13}$ Research from our group showed that Vitamin D supplementation significantly increases serum 25(OH)D concentrations. ${ }^{12}$

Low bone mineral density has been reported in pediatric cancer survivors even several years after completion of cancer therapy. ${ }^{16,17}$ Current national survivorship guidelines ${ }^{18}$ recommend a baseline bone mineral density evaluation followed by laboratory assessments, in order to evaluate bone health beginning two years after therapy completion, particularly for patients with history of acute lymphoblastic leukemia. ${ }^{17,} 18$

Our QI methodology led to successful establishment of the QI team, with specific roles for team members; continued communication of the QI initiative aim and progress to staff; two-way feedback on processes implementation; and accurate and efficient patient identification for data analysis. The EMR-based interventions implemented by the IT team for automated BPA alerts and triggers for Vitamin D laboratory testing and monitoring may have contributed to the observed increase in compliance with the newly developed institutional guideline for Vitamin D testing and supplementation. After conducting educational interventions regarding the standardized criteria for Vitamin D deficiency and insufficiency diagnoses, and the clinical decision-making tree for adequate supplementation, we observed a significant increase in testing and supplementation rates. This finding is consistent with previous studies showing that passive EMR features such as BPA alerts need to be accompanied by complementary strategies such as educational interventions to improve compliance with guidelines and achieve sustainability over time. ${ }^{19}$

Regular feedback about group performance, compared to prior performance were positively received, and helped motivate oncology providers to further adhere to the newly implemented practices. Although additional research is needed to establish the benefit of performance feedback (individual or group) in improving guideline compliance and sustained culture change, our findings support previous studies showing the positive impact of providing timely feedback on improving clinical practice and EMR documentation. ${ }^{20}$ Additionally, hospital leadership support and awareness of the clinical need, and frequent communication with key stakeholders contributed to achieving and sustaining our aim.

There are several limitations in our project to be discussed. First, we focused on one aspect of clinical care in a specific setting. Second, we reviewed data for three months prior to our interventions on a small group of patients. Studying pre-intervention data in a larger group and over a longer period may help to determine temporal variation. Third, our EMR interventions were geared toward BPA alerts and automated testing options in our admission and chemotherapy order sets, which are specific components of our EMR and may not be reproducible in other practices that do not use a similar EMR. However, as most centers 
managing pediatric cancer have had EMRs in place for many years, this limitation should be resolvable. While motivations for performing testing or prescribing supplementation were not explored, providers' perceived utility of the decision-making tree and automated triggers, and positive feedback of frequent reports and reminders, contributed to steady improvement over the first 4 months of the QI initiative and sustainability for 24 months. Additionally, our QI initiative could have been further strengthened by assessing balancing measures to determine whether there were unintended consequences in other parts of the clinical workflow as a result of our QI initiative, such as documentation of time spent by the provider reviewing the clinical decision-making tree, ordering tests and prescribing supplementation.

\section{Conclusion}

Our inter-professional QI initiative, which incorporated automated tools within the EMR, demonstrated success in improving Vitamin D testing and supplementation in children with newly-diagnosed cancer in a large tertiary academic institution with a high prevalence of Vitamin D deficiency and insufficiency. This QI approach may be replicated in similar settings. Future PDSA cycles will address patient compliance with Vitamin D supplementation. QI methodology will continue to be used in the future to improve adherence to other testing and medication prescribing procedures for children with cancer at our institution.

\section{Conflict of Interest statement}

This project was partially supported by the National Cancer Institute K08 CA230306 (Aristizabal) and Dedman Family (Shliakhtsitsava). The content is solely the responsibility of the authors and does not represent the official views of the National Cancer Institute or the National Institutes of Health.

\section{Acknowledgements}

We thank the pediatric hematology/oncology team and the Quality Improvement Committee (Elizabeth Sheldon RN, Lindsay Colliton RN, Sally Steiner RN, Margaret Fitzgerald RN, Teresa Cassidy RN and Stacey Brown RN) at the Peckham Center for Cancer and Blood Disorders at Rady Children's Hospital. The content is solely the responsibility of the authors. We thank Adam Felton for Electronic Medical Record and $\mathrm{EPIC}^{(\mathrm{r})}$ support.

\section{References}

1. Ross AC, Manson JE, Abrams SA, et al. The 2011 report on dietary reference intakes for calcium and vitamin D from the Institute of Medicine: what clinicians need to know. J Clin Endocrinol Metab . Jan 2011;96(1):53-8. doi:10.1210/jc.2010-2704

2. Medicine Io. Conclusions about Vitamin D deficiency in the US and Canada. 2011.

3. Heath AK, Kim IY, Hodge AM, English DR, Muller DC. Vitamin D Status and Mortality: A Systematic Review of Observational Studies. Int J Environ Res Public Health . 01 2019;16(3)doi:10.3390/ijerph16030383

4. Boscoe FP, Schymura MJ. Solar ultraviolet-B exposure and cancer incidence and mortality in the United States, 1993-2002. BMC Cancer . Nov 2006;6:264. doi:10.1186/1471-2407-6-264

5. Holick MF. Vitamin D deficiency. N Engl J Med. Jul 2007;357(3):266-81. doi:10.1056/NEJMra070553

6. Christakos S, Hewison M, Gardner DG, et al. Vitamin D: beyond bone.Ann N Y Acad Sci . May 2013;1287:45-58. doi:10.1111/nyas.12129

7. Cuomo RE, Garland CF, Gorham ED, Mohr SB. Low Cloud Cover-Adjusted Ultraviolet B Irradiance Is Associated with High Incidence Rates of Leukemia: Study of 172 Countries. PLoS One . 2015;10(12):e0144308. doi:10.1371/journal.pone.0144308

8. Borchmann S, Cirillo M, Goergen H, et al. Pretreatment Vitamin D Deficiency Is Associated With Impaired Progression-Free and Overall Survival in Hodgkin Lymphoma. J Clin Oncol . 12 2019;37(36):35283537. doi:10.1200/JCO.19.00985 
9. Cummings EA, Ma J, Fernandez CV, et al. Incident Vertebral Fractures in Children With Leukemia During the Four Years Following Diagnosis.J Clin Endocrinol Metab . Sep 2015;100(9):3408-17. doi:10.1210/JC.2015-2176

10. Halton J, Gaboury I, Grant R, et al. Advanced vertebral fracture among newly diagnosed children with acute lymphoblastic leukemia: results of the Canadian Steroid-Associated Osteoporosis in the Pediatric Population (STOPP) research program. J Bone Miner Res . Jul 2009;24(7):1326-34. doi:10.1359/jbmr.090202

11. Wallace G, Jodele S, Howell J, et al. Vitamin D Deficiency and Survival in Children after Hematopoietic Stem Cell Transplant.Biol Blood Marrow Transplant . Sep 2015;21(9):1627-31. doi:10.1016/j.bbmt.2015.06.009

12. Aristizabal P, Sherer M, Perdomo BP, et al. Sociodemographic and clinical characteristics associated with vitamin D status in newly diagnosed pediatric cancer patients. Pediatr Hematol Oncol . May 2020;37(4):314325. doi: $10.1080 / 08880018.2020 .1721629$

13. Holick MF, Binkley NC, Bischoff-Ferrari HA, et al. Evaluation, treatment, and prevention of vitamin D deficiency: an Endocrine Society clinical practice guideline. J Clin Endocrinol Metab . Jul 2011;96(7):191130. doi:10.1210/jc.2011-0385

14. al Le. The improvement guide: a practical approach to enhancing organization performance. 2 edition ed. Jossey-Bass; 2009.

15. Kurtin P, Stucky E. Standardize to excellence: improving the quality and safety of care with clinical pathways. Pediatr Clin North Am . Aug 2009;56(4):893-904. doi:10.1016/j.pcl.2009.05.005

16. Makitie O, Heikkinen R, Toiviainen-Salo S, Henriksson M, Puukko-Viertomies LR, Jahnukainen K. Longterm skeletal consequences of childhood acute lymphoblastic leukemia in adult males: a cohort study.Eur $J$ Endocrinol . Feb 2013;168(2):281-8. doi:10.1530/EJE-12-0702

17. Marcucci G, Beltrami G, Tamburini A, et al. Bone health in childhood cancer: review of the literature and recommendations for the management of bone health in childhood cancer survivors. Ann Oncol . 06 2019;30(6):908-920. doi:10.1093/annonc/mdz120

18. Group CO. Long-term follow up guidelines for survivors of childhood, adolescent and young adult cancers. http://www.survivorshipguidelines.org/pdf/2018/COG_LTFU_Guidelines_v5.pdf

19. Smith AJ, Skow A, Bodurtha J, Kinra S. Health information technology in screening and treatment of child obesity: a systematic review.Pediatrics . Mar 2013;131(3):e894-902. doi:10.1542/peds.2012-2011

20. Opila DA. The impact of feedback to medical housestaff on chart documentation and quality of care in the outpatient setting. J Gen Intern Med . Jun 1997;12(6):352-6. doi:10.1046/j.1525-1497.1997.00059.x

\section{Legend}

FIGURE 1 Ishikawa diagram.

FIGURE 2 Key driver diagram.

FIGURE 3 Decision-Making Tree.

FIGURE 4 Vitamin D testing rates. P-chart with vitamin D testing and annotated improvement interventions in orange. Desired direction of change is noted in bold green arrow. GD Guideline development; E, Education; DT, Decision making tree; BPA, Best Practice Alert; CL, centerline; LCL, lower control limit; UCL, upper control limit. Plan-Do-Study-Act (PDSA) Cycle 1= GD and DT development; Cycle $2=\mathrm{E}$, DT implementation, Electronic Medical Record (EMR) triggers (BPA).

FIGURE 5 Vitamin D supplementation and follow-up post-supplementation rates. P-chart with vitamin D supplementation. Desired direction of change is noted in bold green arrow. E, Education; DT, Decision making tree; BPA, Best Practice Alert; CL, centerline; LCL, lower control limit; UCL, upper control limit. 
Plan-Do-Study-Act (PDSA) Cycle 2 = E, DT implementation, Electronic Medical Record (EMR) triggers (BPA).

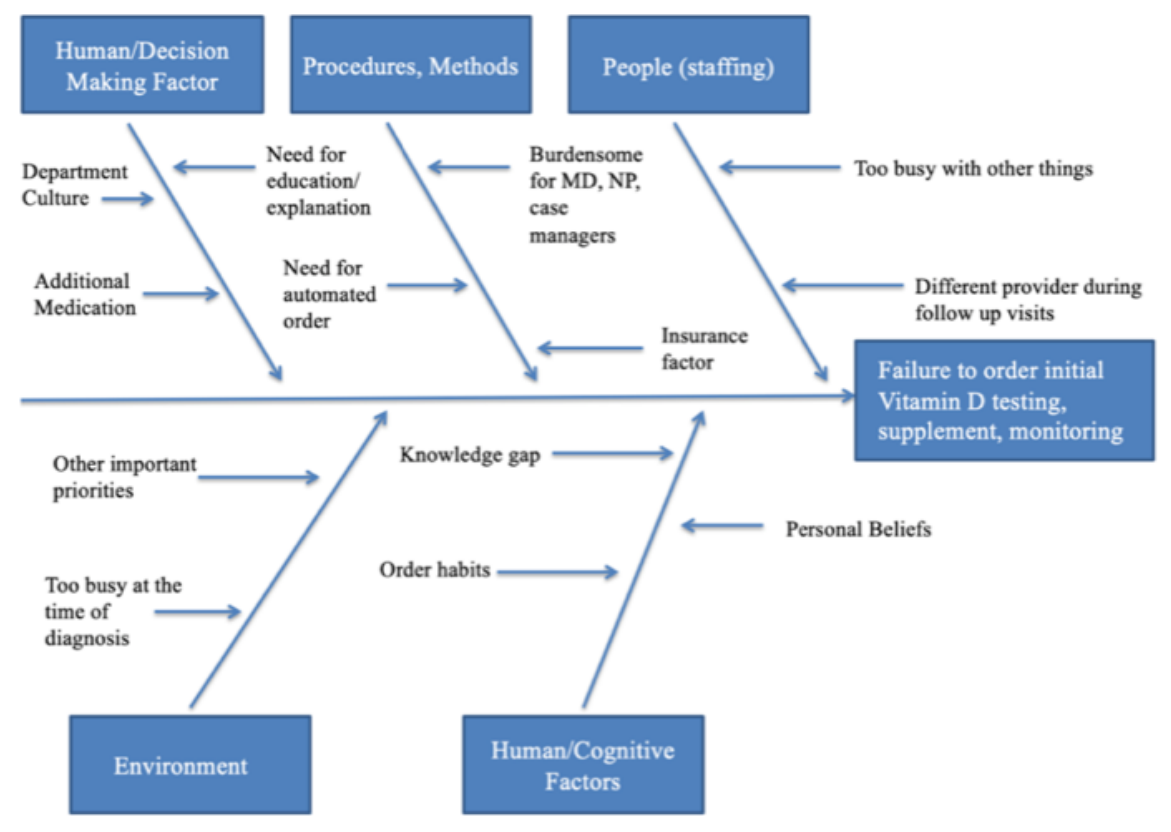

\begin{tabular}{|c|l|}
\hline SMART Aim & Primary Drivers \\
\hline $\begin{array}{c}\text { In } 6 \text { months, to improve } \\
\text { Vitamin } \mathrm{D} \text { testing and } \\
\text { supplementation among } \\
\text { children (ages } 2 \text {-18 years) } \\
\text { with newly diagnosed } \\
\text { cancer to } \geq 80 \%\end{array}$ \\
$\begin{array}{c}\text { EHR and best } \\
\text { practices integration }\end{array}$ \\
$\begin{array}{c}\text { Systems and } \\
\text { workflow }\end{array}$ \\
\hline $\begin{array}{c}\text { Leadership } \\
\text { and Buy-in }\end{array}$ \\
Awareness
\end{tabular}



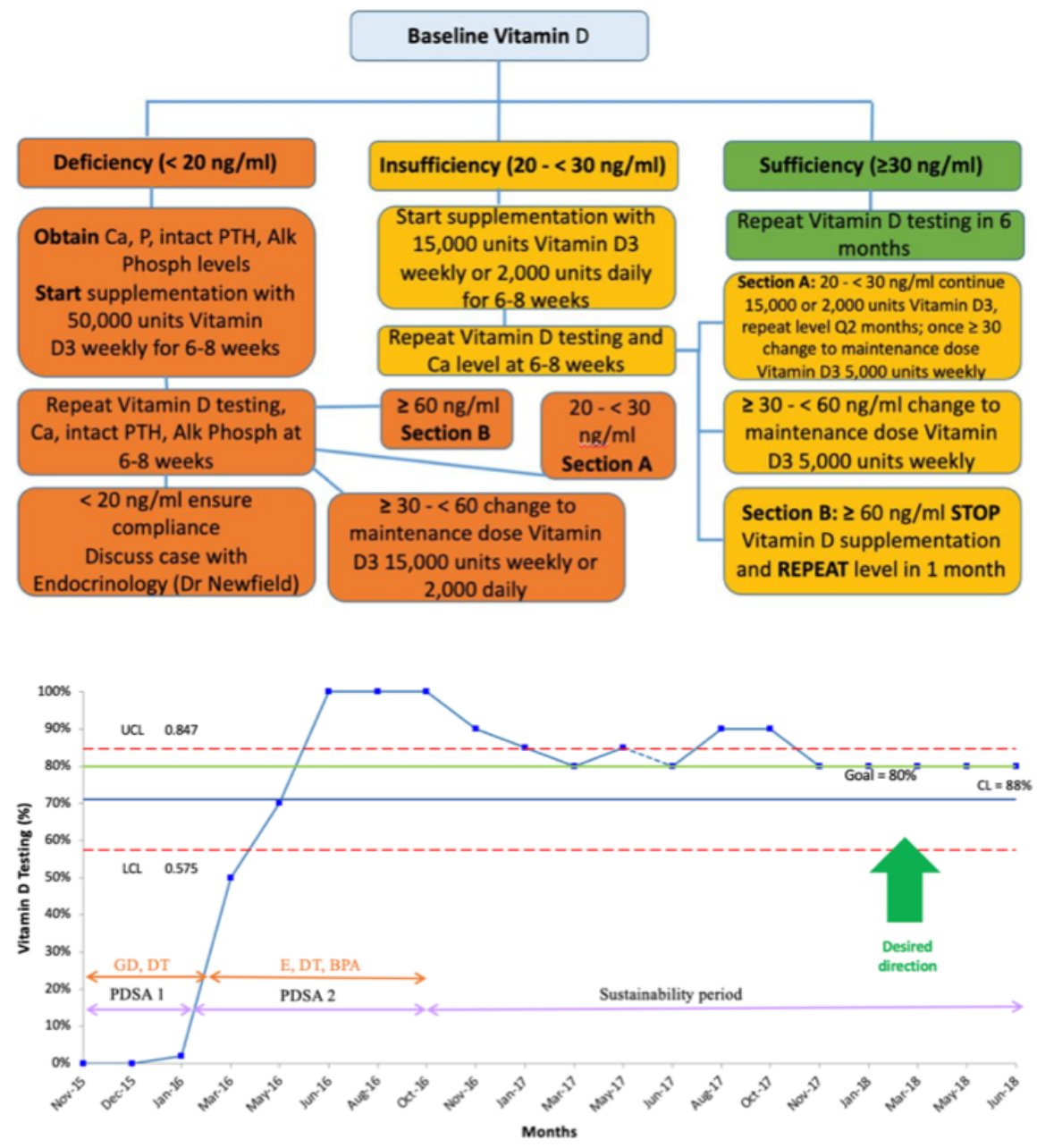

Vitamin D Supplementation

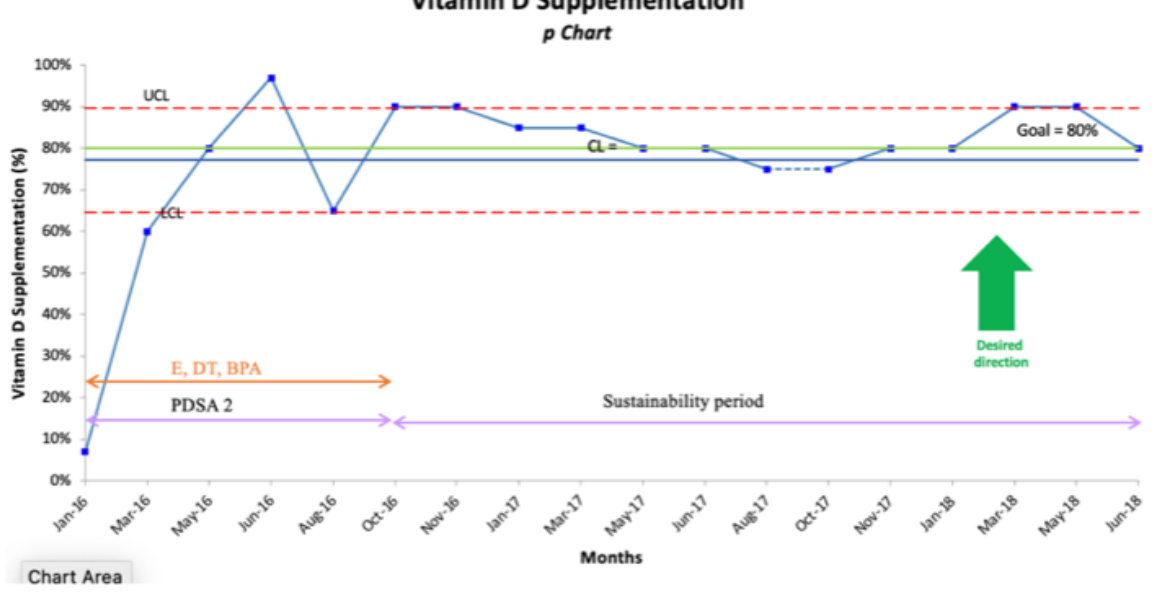

